\title{
A Generalization on Some New Types of Hardy-Hilbert's Integral Inequalities
}

\author{
Banyat Sroysang \\ Department of Mathematics and Statistics, Faculty of Science and Technology, Thammasat University, Pathum Thani 12121, Thailand \\ Correspondence should be addressed to Banyat Sroysang; banyat@mathstat.sci.tu.ac.th
}

Received 15 May 2013; Accepted 17 September 2013

Academic Editor: Wilfredo Urbina

Copyright (c) 2013 Banyat Sroysang. This is an open access article distributed under the Creative Commons Attribution License, which permits unrestricted use, distribution, and reproduction in any medium, provided the original work is properly cited.

Sulaiman presented, in 2008, new kinds of Hardy-Hilbert's integral inequality in which the weight function is homogeneous. In this paper, we present a generalization on the kinds of Hardy-Hilbert's integral inequality.

\section{Introduction and Preliminaries}

For any two nonnegative measurable functions $f$ and $g$ such that

$$
0<\int_{0}^{\infty} f^{2}(x) d x<\infty, \quad 0<\int_{0}^{\infty} g^{2}(y) d y<\infty,
$$

we have the Hilbert's integral inequality [1] that

$$
\begin{aligned}
& \iint_{0}^{\infty} \frac{f(x) g(y)}{x+y} d x d y \\
& \quad<\pi\left(\int_{0}^{\infty} f^{2}(x) d x \int_{0}^{\infty} g^{2}(y) d y\right)^{1 / 2} .
\end{aligned}
$$

The constant $\pi$ is the best possible. In 1925, Hardy [2] extended the Hilbert's integral inequality into the integral inequality as follows. If $p>1,1 / p+1 / q=1$, and $f, g \geq 0$ such that

$$
0<\int_{0}^{\infty} f^{p}(x) d x<\infty, \quad 0<\int_{0}^{\infty} g^{q}(y) d y<\infty,
$$

then we have the Hardy-Hilbert's integral inequality that

$$
\begin{aligned}
& \iint_{0}^{\infty} \frac{f(x) g(y)}{x+y} d x d y \\
& \quad<\frac{\pi}{\sin (\pi / p)}\left(\int_{0}^{\infty} f^{p}(x) d x\right)^{1 / p}\left(\int_{0}^{\infty} g^{q}(y) d y\right)^{1 / q} .
\end{aligned}
$$

The constant $\pi / \sin (\pi / p)$ is the best possible. Both the two inequalities are important in mathematical analysis and its applications [3].

In 1938, Widder [4] studied on the Stieltjes Transform $S_{f}(y)=\int_{0}^{\infty} f(x) /(x+y) d x$.

Now, we recall the beta function $B$ as follows:

$$
B(p, q)=\int_{0}^{1} t^{p-1}(1-t)^{q-1} d t, \quad \text { where } p, q>0 .
$$

In 2001, Yang [5] extended the Hardy-Hilbert's integral inequality into the following integral inequality. If $p, q>0$, $\lambda>2-\min \{p, q\}, 1 / p+1 / q=1$, and $f, g \geq 0$ such that

$$
\begin{aligned}
& 0<\int_{0}^{\infty} x^{1-\lambda} f^{p}(x) d x<\infty, \\
& 0<\int_{0}^{\infty} y^{1-\lambda} g^{q}(y) d y<\infty,
\end{aligned}
$$

then we have

$$
\begin{aligned}
& \iint_{0}^{\infty} \frac{f(x) g(y)}{(x+y)^{\lambda}} d x d y \\
& \quad<k_{\lambda}(p)\left(\int_{0}^{\infty} x^{1-\lambda} f^{p}(x) d x\right)^{1 / p}\left(\int_{0}^{\infty} y^{1-\lambda} g^{q}(y) d y\right)^{1 / q},
\end{aligned}
$$

where $k_{\lambda}(p)=B(1+(\lambda-2) / p, 1+(\lambda-2) / p)$. The constant $k_{\lambda}(p)$ is the best possible. 
We also recall that a nonnegative function $f(x, y)$ which is said to be homogeneous function of degree $\lambda$ if $f(t x, t y)=$ $t^{\lambda} f(x, y)$ for all $t>0$. And we say that $K(u, v)$ is increasing if $K(1, t)$ and $K(t, 1)$ are increasing functions.

In 2008, Sulaiman [6] gave new integral inequality similar to the Hardy-Hilbert's integral inequality. If $a, b>0, p>1$, $1 / p+1 / q=1,0<\lambda \leq \min \{(1-b) p / q,(1-a) q / p\}, K(u, v)$ is a positive increasing homogeneous function of degree $\lambda$, and $f, g \geq 0$ and

$$
F(x)=\int_{0}^{x} f(t) d t, \quad G(x)=\int_{0}^{x} g(t) d t \quad \forall x>0,
$$

then, for all $T>0$, we have

$$
\begin{aligned}
& \iint_{0}^{T} \frac{F(u) G(v)}{K(u, v)} d u d v \\
& \leq T^{\alpha} \sqrt[p]{p K_{1}} \sqrt[q]{q K_{2}}\left(\int_{0}^{T}(T-t) F^{p-1}(t) f(t) d t\right)^{1 / p} \\
& \quad \times\left(\int_{0}^{T}(T-t) G^{q-1}(t) g(t) d t\right)^{1 / q},
\end{aligned}
$$

where

$$
K_{1}=\int_{0}^{1} \frac{t^{a-1}}{K(1, t)} d t, \quad K_{2}=\int_{0}^{1} \frac{t^{b-1}}{K(t, 1)} d t
$$

In this paper, we present a generalization of the integral inequality (9) and its applications. Next proposition will be used in the next section.

Proposition 1 (see [6]). Let $g$ be a positive increasing function, and $a, b>0$. Then, for all $x \geq 1$, one has

$$
x^{-a} \int_{0}^{x} \frac{t^{a-1}}{g(t)} d t \leq \int_{0}^{1} \frac{t^{a-1}}{g(t)} d t
$$

\section{Main Results}

Theorem 2. Let $0<a, b<1<p, 1 / p+1 / q=1,0<\lambda \leq$ $\min \{(1-b) p / q,(1-a) q / p\}$, and let $K(u, v)$ be positive increasing homogeneous function of degree $\lambda$, and $f, g \geq 0$ and

$$
F(x)=\int_{0}^{x} f(t) d t, \quad G(x)=\int_{0}^{x} g(t) d t \quad \forall x>0,
$$

and let $\psi$ be a function such that $\psi(x) \geq x$ for all $x>0$.

Then, for all $T>0$, one has

$$
\begin{aligned}
& \iint_{0}^{T} \frac{F(u) G(v)}{\psi(K(u, v))} d u d v \\
& \leq T^{1-\lambda} \sqrt[p]{p K_{1}} \sqrt[q]{q K_{2}}\left(\int_{0}^{T}(T-t) F^{p-1}(t) f(t) d t\right)^{1 / p} \\
& \quad \times\left(\int_{0}^{T}(T-t) G^{q-1}(t) g(t) d t\right)^{1 / q},
\end{aligned}
$$

where

$$
K_{1}=\int_{0}^{1} \frac{t^{a-1}}{K(1, t)} d t, \quad K_{2}=\int_{0}^{1} \frac{t^{b-1}}{K(t, 1)} d t
$$

Proof. Let $T>0$ and $M=\iint_{0}^{T} F(u) G(v) / \psi(K(u, v)) d u d v$.

By the Hölder inequality, the assumption of $\psi$, and the Tonelli theorem, we have

$$
\begin{aligned}
& M= \iint_{0}^{T} \frac{F(u) v^{(a-1) / p}}{u^{(b-1) / q} \psi^{1 / p}(K(u, v))} \\
& \times \frac{G(v) u^{(b-1) / q}}{v^{(a-1) / p} \psi^{1 / q}(K(u, v))} d u d v \\
& \leq\left(\iint_{0}^{T} \frac{F^{p}(u) v^{a-1}}{u^{(b-1) p / q} \psi(K(u, v))} d u d v\right)^{1 / p} \\
& \times\left(\int_{0}^{T} \frac{G^{q}(v) u^{b-1}}{v^{(a-1) q / p} \psi(K(u, v))} d u d v\right)^{1 / q} \\
&=\left(\int_{0}^{T} F^{p}(u) \int_{0}^{T} \frac{u^{(1-b) p / q} v^{a-1}}{\psi(K(u, v))} d v d u\right)^{1 / p} \\
& \times\left(\int_{0}^{T} G^{q}(v) \int_{0}^{T} \frac{u^{b-1} v^{(1-a) q / p}}{\psi(K(u, v))} d u d v\right)^{1 / q} \\
& \leq\left(\int_{0}^{T} F^{p}(u) \int_{0}^{T} \frac{u^{(1-b) p / q} v^{a-1}}{K(u, v)} d v d u\right)^{1 / p} \\
& \times\left(\int_{0}^{T} G^{q}(v) \int_{0}^{T} \frac{u^{b-1} v^{(1-a) q / p}}{K(u, v)} d u d v\right)^{1 / q} \\
&\left.u^{q}(v) \int_{0}^{T} \frac{v^{(1-b)(p / q)+a-1}(v / u)^{a-1}}{u^{\lambda}(1, v / u)} d v d u\right)^{1 / p}
\end{aligned}
$$

Now, we put $t=v / u$ and $d t=d v / u$ for the first integral, and then we put $t=u / v$ and $d t=d u / v$ for the second integral.

And, by Proposition 1, one has

$$
\begin{aligned}
M \leq & \left(\int_{0}^{T} u^{a+(1-b)(p / q)-\lambda} F^{p}(u) \int_{0}^{T / u} \frac{t^{a-1}}{K(1, t)} d t d u\right)^{1 / p} \\
& \times\left(\int_{0}^{T} v^{b+(1-a)(q / p)-\lambda} G^{q}(v) \int_{0}^{T / u} \frac{t^{b-1}}{K(t, 1)} d t d v\right)^{1 / q}
\end{aligned}
$$




$$
\begin{aligned}
= & \left(T^{a+(1-b)(p / q)-\lambda} \int_{0}^{T} F^{p}(u)\left(\frac{u}{T}\right)^{a+(1-b)(p / q)-\lambda}\right. \\
& \left.\times \int_{0}^{T / u} \frac{t^{a-1}}{K(1, t)} d t d u\right)^{1 / p} \\
& \times\left(T^{b+(1-a)(q / p)-\lambda} \int_{0}^{T} G^{q}(v)\left(\frac{v}{T}\right)^{b+(1-a)(q / p)-\lambda}\right. \\
& \left.\times \int_{0}^{T / u} \frac{t^{b-1}}{K(t, 1)} d t d v\right)^{1 / q} \\
\leq & \times \int_{0}^{a+(1-b)(p / q)-\lambda} \int_{0}^{T} F^{p}(u)\left(\frac{u}{T}\right)^{a} \\
& \times\left(T^{b+(1-a)(q / p)-\lambda} \int_{0}^{T} G^{q}(v)\left(\frac{v}{T}\right)^{b}\right. \\
= & T^{1-\lambda}\left(K_{1} \int_{0}^{T} F^{p}(u) d u\right)^{1 / p} \\
& \times\left(K_{2} \int_{0}^{T} G^{q}(v) d v\right)^{1 / q} \cdot \\
& \left.\left.\times \int_{0}^{T / u} \frac{t^{b-1}}{K(t, 1)} d t d v\right)^{1 / q} G^{q}(v) \int_{0}^{1} \frac{t^{b-1}}{K(t, 1)} d t d v\right)^{1 / q} \\
= & T^{1-\lambda}\left(\int_{0}^{T} F^{p}(u)\left(\frac{u}{T}\right)^{a} \int_{0}^{T / u} \frac{t^{a-1}}{K(1, t)} d t d u\right)^{1 / p} \\
& \times\left(\int_{0}^{T} G^{q}(v)\left(\frac{v}{T}\right)^{b} \int_{0}^{T / u} \frac{t^{b-1}}{K(t, 1)} d t d v\right)^{1 / q}
\end{aligned}
$$

Then, by the assumption, one has

$$
\begin{aligned}
M \leq & T^{1-\lambda}\left(K_{1} \int_{0}^{T} \int_{0}^{u}\left(F^{p}(t)\right)^{\prime} d t d u\right)^{1 / p} \\
& \times\left(K_{2} \int_{0}^{T} \int_{0}^{v}\left(G^{q}(t)\right)^{\prime} d t d v\right)^{1 / q} \\
= & T^{1-\lambda}\left(p K_{1} \int_{0}^{T} \int_{0}^{u} F^{p-1}(t) f(t) d t d u\right)^{1 / p} \\
& \times\left(q K_{2} \int_{0}^{T} \int_{0}^{v} G^{q-1}(t) f(t) d t d v\right)^{1 / q}
\end{aligned}
$$

$$
\begin{aligned}
= & T^{1-\lambda}\left(p K_{1} \int_{0}^{T} F^{p-1}(t) f(t) \int_{t}^{T} d u d t\right)^{1 / p} \\
& \times\left(q K_{2} \int_{0}^{T} G^{q-1}(t) f(t) \int_{t}^{T} d v d t\right)^{1 / q} \\
= & T^{1-\lambda}\left(p K_{1} \int_{0}^{T}(T-t) F^{p-1}(t) f(t) d t\right)^{1 / p} \\
& \times\left(q K_{2} \int_{0}^{T}(T-t) G^{q-1}(t) f(t) d t\right)^{1 / q} \\
= & T^{1-\lambda} \sqrt[p]{p K_{1}} \sqrt[q]{q K_{2}} \\
& \times\left(\int_{0}^{T}(T-t) F^{p-1}(t) f(t) d t\right)^{1 / p} \\
& \times\left(\int_{0}^{T}(T-t) G^{q-1}(t) g(t) d t\right)^{1 / q} .
\end{aligned}
$$

This proof is completed.

\section{Applications}

Corollary 3. Let $0<a, b<1<p, 1 / p+1 / q=1$ and $0<$ $\lambda \leq \min \{(1-b) p / q,(1-a) q / p\}$, and let $K(u, v)$ be a positive increasing homogeneous function of degree $\lambda$, and $f, g \geq 0$ and $F(x)=\int_{0}^{x} f(t) d t, \quad G(x)=\int_{0}^{x} g(t) d t \quad \forall x>0$.

Then, for all $T>0$, one has

(a) $\iint_{0}^{T} \frac{F(u) G(v)}{K(u, v)} d u d v$

$$
\begin{aligned}
\leq & T^{1-\lambda} \sqrt[p]{p K_{1}} \sqrt[q]{q K_{2}} \\
& \times\left(\int_{0}^{T}(T-t) F^{p-1}(t) f(t) d t\right)^{1 / p}
\end{aligned}
$$

$$
\times\left(\int_{0}^{T}(T-t) G^{q-1}(t) g(t) d t\right)^{1 / q},
$$

(b) $\iint_{0}^{T} \frac{F(u) G(v)}{1+K(u, v)} d u d v$

$$
\begin{aligned}
\leq & T^{1-\lambda} \sqrt[p]{p K_{1}} \sqrt[q]{q K_{2}} \\
& \times\left(\int_{0}^{T}(T-t) F^{p-1}(t) f(t) d t\right)^{1 / p} \\
& \times\left(\int_{0}^{T}(T-t) G^{q-1}(t) g(t) d t\right)^{1 / q},
\end{aligned}
$$




$$
\text { (c) } \begin{aligned}
\iint_{0}^{T} \frac{F(u) G(v)}{(1+K(u, v)) K(u, v)} d u d v \\
\leq T^{1-\lambda} \sqrt[p]{p K_{1} \sqrt[q]{q K_{2}}} \\
\quad \times\left(\int_{0}^{T}(T-t) F^{p-1}(t) f(t) d t\right)^{1 / p} \\
\quad \times\left(\int_{0}^{T}(T-t) G^{q-1}(t) g(t) d t\right)^{1 / q}
\end{aligned}
$$

(d) $\iint_{0}^{T} \frac{F(u) G(v)}{e^{K(u, v)}} d u d v$

$$
\begin{aligned}
\leq & T^{1-\lambda} \sqrt[p]{p K_{1}} \sqrt[q]{q K_{2}}\left(\int_{0}^{T}(T-t) F^{p-1}(t) f(t) d t\right)^{1 / p} \\
& \times\left(\int_{0}^{T}(T-t) G^{q-1}(t) g(t) d t\right)^{1 / q}
\end{aligned}
$$

where

$$
K_{1}=\int_{0}^{1} \frac{t^{a-1}}{K(1, t)} d t, \quad K_{2}=\int_{0}^{1} \frac{t^{b-1}}{K(t, 1)} d t .
$$

Proof. (a) This follows from Theorem 2 where $\psi(x)=x$ for all $x$.

(b) This follows from Theorem 2 where $\psi(x)=1+x$ for all $x$.

(c) This follows from Theorem 2 where $\psi(x)=x+x^{2}$ for all $x$.

(d) This follows from Theorem 2 where $\psi(x)=e^{x}$ for all $x$.

\section{Open Problem}

In this section, we pose a question that is how to generalize the integral inequality (13) if $\psi$ may not satisfy the property $\psi(x) \geq x$ for all $x>0$.

\section{Acknowledgments}

The author would like to thank the referees for their useful comments and suggestions.

\section{References}

[1] G. H. Hardy, J. E. Littlewood, and G. Pólya, Inequalities, Cambridge University Press, Cambridge, UK, 2nd edition, 1952.

[2] G. H. Hardy, "Notes on a theorem of Hilbert concerning series of positive terms," Proceedings of the London Mathematical Society, vol. 23, pp. 45-46, 1925.

[3] D. S. Mitrinović, J. E. Pečarić, and A. M. Fink, Inequalities Involving Functions and Their Integrals and Derivatives, vol. 53 of Mathematics and Its Applications (East European Series), Kluwer Academic Publishers, Dordrecht, The Netherlands, 1991.
[4] D. V. Widder, "The Stieltjes transform," Transactions of the American Mathematical Society, vol. 43, no. 1, pp. 7-60, 1938.

[5] B. Yang, "On Hardy-Hilbert's integral inequality," Journal of Mathematical Analysis and Applications, vol. 261, pp. 295-306, 2001.

[6] W. T. Sulaiman, "A study on some new types of Hardy-Hilbert's integral inequalities," Banach Journal of Mathematical Analysis, vol. 2, no. 1, pp. 16-20, 2008. 


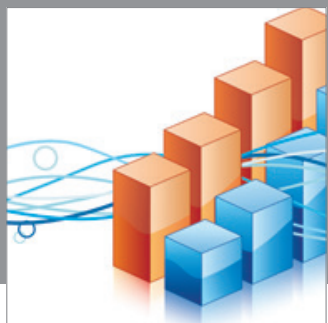

Advances in

Operations Research

mansans

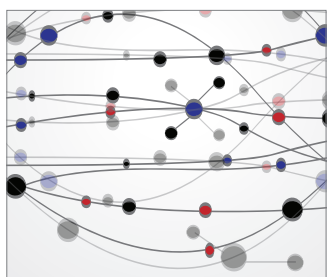

The Scientific World Journal



Submit your manuscripts at http://www.hindawi.com


Journal of

Function Spaces

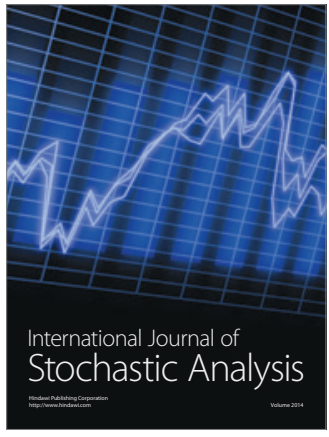

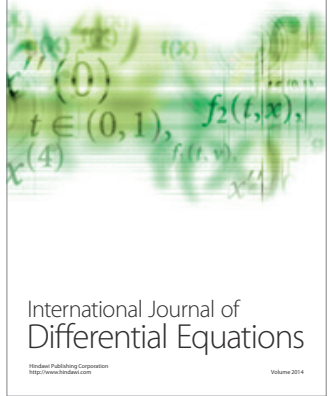
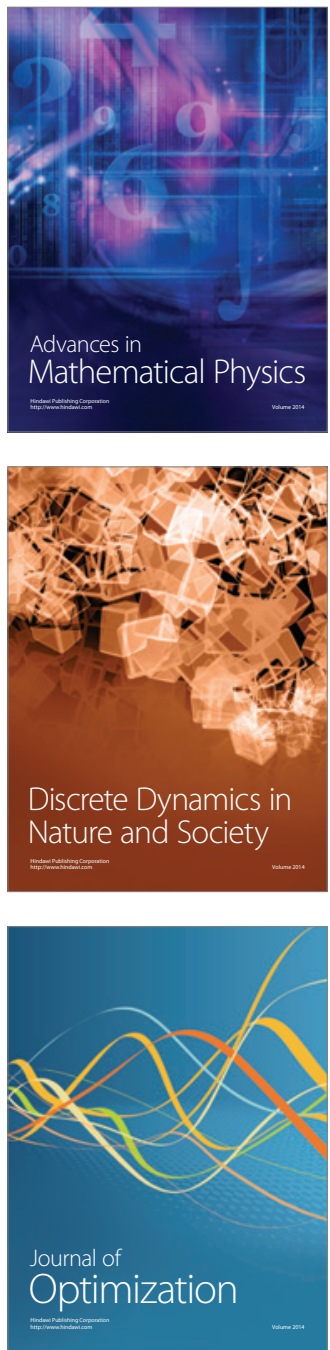